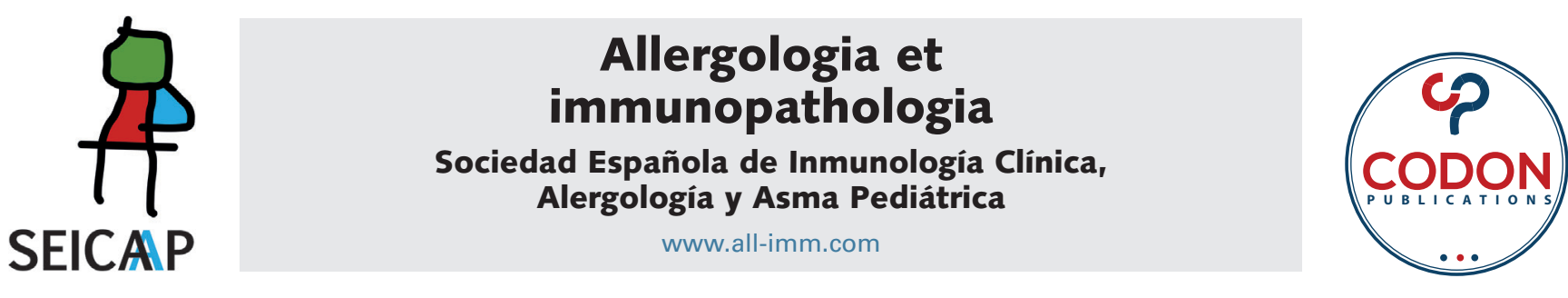

\title{
Transient increased immunoglobulin levels in a hyper-IgM syndrome patient with COVID-19 infection
}

\author{
Molood Safarirada, Ali Abbaszadeh Ganjib, Farzad Nazaric,d, Reza Yazdanic, \\ Hassan Abolhassanic,e, Ahmad Vosughi Motlagha*
}

\author{
${ }^{a}$ Department of Pediatrics, North Khorasan University of Medical Sciences, Bojnurd, Iran \\ ${ }^{b}$ Research Committee, North Khorasan University of Medical Sciences, Bojnurd, Iran \\ 'Research Center for Immunodeficiencies, Pediatrics Center of Excellence, Children's Medical Center, Tehran University of Medical \\ Science, Tehran, Iran \\ 'Immunology Today, Universal Scientific Education and Research Network (USERN), Tehran, Iran \\ eDivision of Clinical Immunology, Department of Laboratory Medicine, Karolinska Institute at Karolinska University Hospital \\ Huddinge, Stockholm, Sweden
}

Received 22 June 2021; Accepted 19 October 2021

Available online 1 November 2021

\section{KEYWORDS \\ COVID-19; \\ humoral immunity; hyper-lgM syndrome; IgG; primary immunodeficiency}

\begin{abstract}
Background: The coronavirus disease 2019 (COVID-19) pandemic has affected millions of people around the world. This zoonotic-enveloped virus is primarily transmitted through inhalation. Infected people are commonly asymptomatic or manifest mild symptoms, including fever, cough, diarrhea, and fatigue. However, it may lead to severe patterns associated with multiple organ failure in individuals with an impaired immune system.

Objective: Here we report a 7-year-old girl with hyper-immunoglobulin M (IgM) (HIgM) phenotype, admitted to the hospital emergency department with fever, cough, and pneumonia symptoms because of the COVID-19 infection. Coronavirus infection was confirmed by a positive real-time polymerase chain reaction test. Surprisingly, serum levels of both IgG and IgA of the patient were transiently normalized during the COVID-19 infection when tested prior to the monthly injection of intravenous immunoglobulin. After she recovered from the COVID infection, her immunoglobulin levels returned to the primary stage and she demonstrated HIgM phenotype.

Conclusion: Since this transient increase in the levels of immunoglobulins was solely observed during the COVID-19 infection, and no other infectious episodes were diagnosed in the patient, clarifying the exact cause would help to understand in a better manner the implications and specification of humoral immunity in patients with primary antibody deficiencies.

(c) 2021 Codon Publications. Published by Codon Publications.
\end{abstract}

*Corresponding author: Ahmad Vosughi Motlagh, MD, Department of Pediatrics, North Khorasan University of Medical Sciences, Bojnurd, Iran. Email address: dr.ahmadvosughi@yahoo.com 


\section{Introduction}

In the winter of 2019, the spread of severe acute respiratory syndrome-coronavirus 2 (SARS-CoV-2) led to the coronavirus disease 2019 (COVID-19) pandemic, affecting millions of people around the world. During this pandemic, people with preexisting medical conditions were at a higher risk of mortality and morbidity. It has been suggested that probably its origin was zoonotic, and was primarily transmitted through inhalation; hence, using a face mask and isolation are the best means to prevent its spread. ${ }^{1}$

The most common symptoms of the COVID-19 infection are fever, cough, sore throat, dyspnea, fatigue, diarrhea, and malaise. Most of the infected patients were asymptomatic or manifested a mild disease; however, it could lead to pneumonia, acute respiratory distress syndrome (ARDS), and multiple organ failure such as acute kidney disease, myocarditis, and heart failure in some patients (especially the elderly patients and those with comorbidities). ${ }^{2-7}$ Laboratory findings in infected patients revealed hypoalbuminemia as well as increase in fibrin degradation product (D-dimer), C-reactive protein (CRP), lactate dehydrogenase (LDH), and erythrocyte sedimentation rate (ESR). ${ }^{8,9}$ Definitive diagnosis is made by real-time polymerase chain reaction (RT-PCR), computed tomography scan (CT scan), or serology tests using the chemiluminescence immunoassay technique. ${ }^{10-14}$ To date, no specific treatment has proved to be curative, but some studies have shown that use of tocilizumab ${ }^{15}$ or convalescent plasma transfusion, ${ }^{16}$ could be beneficial.

Owing to the challenge in the severity of infection in immunocompromised patients and the lack of efficient treatment for this category of patients, ${ }^{1}$ collective information on diagnosis and management of these patients are important for estimation of prognosis. Herein, we report a patient with hyper-immunoglobulin M (IgM) (HIgM) syndrome, who demonstrated transient normalization of humoral immunity during the COVID-19 infection.

\section{Case Presentation}

A 7-year-old girl from consanguineous healthy parents was born through a full-term, normal, natural vaginal delivery. There was no history of primary immunodeficiency disorders (PIDs) in her family. She was growing normally with no vaccination side effects until she was 1 year old. At the age of 1 year, she manifested recurrent fever, upper respiratory tract infections, and otitis media. During the second year of her life, she developed severe pneumonia, which improved after broad-spectrum antibiotic treatment.

At the age of 4 years, the patient demonstrated failure to thrive (FTT) syndrome, presenting with low stature and weight-for-age percentiles. At this age, she developed osteomyelitis of the hip and was hospitalized. In the general examination, FTT, huge splenomegaly, anemia, and thrombocytopenia were determined. She had a hip capsule biopsy, which revealed granulomatous lesion. The patient also underwent an endoscopic check-up for celiac disease, which was ruled out, but a mild inflammation was reported in her duodenum. Her leishmaniasis test was negative. Evaluation of levels of serum immunoglobulins demonstrated a high IgM level, while IgA and IgG levels were within normal range (Figure 1). She was diagnosed with HlgM syndrome and immunoglobulin replacement therapy was initiated.

Two years later, she was re-examined for another episode of infection, and the HIgM diagnosis was again confirmed. The patient also demonstrated a reduced level (50\%) of hemolytic complement activity in the laboratory

\section{$\mathrm{mg} / \mathrm{dl}$}

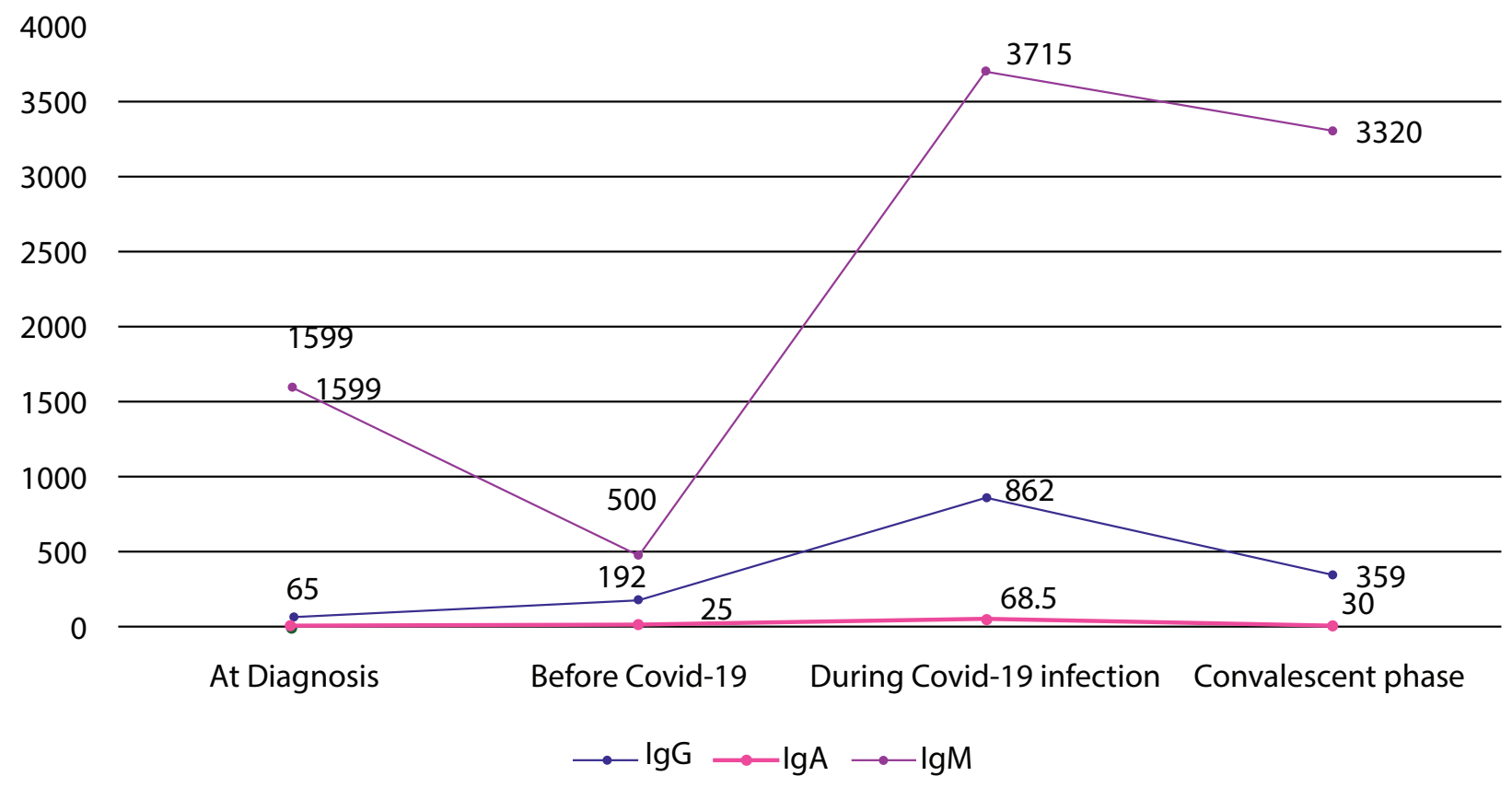

Figure 1 Normalization of IgG and IgA level in patient with Hyper IgM syndrome during COVID-19 infection without Ig replacement therapy. 
Table 1 Immunologic profile of hyper-lgM syndrome patient with COVID-19 infection.

\begin{tabular}{lcc}
\hline Test & Result & $\begin{array}{c}\text { Normal range } \\
\text { for age (years) }\end{array}$ \\
\hline White blood cells $\left(10^{3}\right)$ & 5.8 & $4-11$ \\
RBC $\left(10^{6} / \mu \mathrm{L}\right)$ & 3.27 & $4.2-6.3$ \\
$\mathrm{Hb}(\mathrm{g} / \mathrm{dL})$ & 7.9 & $12-16$ \\
$\mathrm{Hct}(\%)$ & 22.6 & $30-45$ \\
MCV $(\mathrm{fl})$ & 69.1 & $80-100$ \\
MCH $(\mathrm{pg})$ & 24.2 & $27-32$ \\
MCHC (g/dL) & 35.0 & $33-38$ \\
Platelets $\left(10^{3} / \mu \mathrm{L}\right)$ & 74 & $150-450$ \\
Neutrophil $(\%)$ & 70 & $20-45$ \\
Lymphocyte $(\%)$ & 22 & $46-76$ \\
ESR, $1 \mathrm{~h}(\mathrm{~mm} / \mathrm{h})$ & 140.0 & $<50$ \\
HIV Ab (index) & 0.3 & $<1$ \\
CH50 (\%) & 8 & $50-150$ \\
\hline
\end{tabular}

ESR: erythrocyte sedimentation rate; $\mathrm{Hb}$ : hemoglobin; Hct: hematocrit; HIV Ab: human immunodeficiency virus antibodies; $\mathrm{MCH}$ : mean corpuscular hemoglobin; MCHC: mean corpuscular hemoglobin concentration; MCV: mean corpuscular volume; RBC: red blood cells

test (CH50 assay) but without any clinical manifestations of autoimmunity (Table 1). Despite the diagnosis of HIgM syndrome, the patient, now 6 years old, had not received any appropriate prophylactic treatment (including prophylactic antibiotics) and regular intravenous immunoglobulin (IVIg) treatment. Again, she was referred to the hospital emergency department with symptoms of fever, cough, and pneumonia. Diagnosis of the COVID-19 infection was confirmed by the RT-PCR and presence of consolidation in her CT scan. After the COVID-19 diagnosis, treatment with ceftriaxone (75 mg/kg/day), vancomycin (10 mg/kg/q $6 \mathrm{~h})$, azithromycin $(10 \mathrm{mg} / \mathrm{kg}$ for the first dose, then $5 \mathrm{mg} / \mathrm{kg}$ / day from the second day), and chloroquine $(10 \mathrm{mg} / \mathrm{kg}$ for the first dose, then $5 \mathrm{mg} / \mathrm{kg} / \mathrm{q} 12 \mathrm{~h}$ ) were administrated. After 2 months, her immunoglobulin levels were checked during the COVID-19 treatment (Figure 1). Surprisingly, the serum IgG and IgA levels were within the normal range despite the COVID-19 infection although she was tested without receiving IVIg and any plasma therapy. Finally, she was discharged after recovering from the COVID-19 infection (Table 2). The patient was evaluated for the COVID-19 infection, and her serology test was negative. Her serum immunoglobulin levels were again tested after recovery, and surprisingly, her immunoglobulins had returned to the pre-COVID-19 infection levels (Figure 1).

\section{Discussion}

We presented a case of 7-year-old girl diagnosed with HIgM syndrome and the COVID-19 infection. The patient had normal IgG, IgA, and IgE levels after having the COVID-19 infection; however, her IgG and IgA levels declined after recovering from the COVID-19 infection.

The HIgM syndrome is characterized by low or undetectable serum IgG, IgA, and IgE with normal or high levels
Table 2 Immunologic profile of hyper-IgM syndrome patient after recovering from COVID-19 infection.

\begin{tabular}{lcc}
\hline Test & Result & $\begin{array}{c}\text { Normal range } \\
\text { for age (years) }\end{array}$ \\
\hline White blood cells $\left(10^{3}\right)$ & 3.89 & $4-11$ \\
RBC $\left(10^{6} / \mu L\right)$ & 4.12 & $4.2-6.3$ \\
Hb $(\mathrm{g} / \mathrm{dL})$ & 9.6 & $12-16$ \\
$\mathrm{Hct}(\%)$ & 30.5 & $30-45$ \\
MCV $(\mathrm{fl})$ & 74 & $80-100$ \\
MCH $(\mathrm{pg})$ & 23.3 & $27-32$ \\
MCHC $(\mathrm{g} / \mathrm{dL})$ & 31.5 & $33-38$ \\
Platelets $\left(10^{3} / \mu \mathrm{L}\right)$ & 136 & $150-450$ \\
Neutrophil $(\%)$ & 59.1 & $20-45$ \\
Lymphocyte $(\%)$ & 23.4 & $46-76$ \\
\hline
\end{tabular}

$\mathrm{Hb}$ : hemoglobin; Hct: hematocrit; $\mathrm{MCH}$ : mean corpuscular hemoglobin; MCHC: mean corpuscular hemoglobin concentration; MCV: mean corpuscular volume; RBC: red blood cells

of serum IgM. The primary difficulty with HIgM patients is the failure of switching of immunoglobulin isotype in B-cells from IgM/IgD to other switched isotypes. ${ }^{16}$ Previous studies have provided evidence that a wide range of immunity could be induced by SARS-CoV-2 in individuals in both humoral and cellular immunity. ${ }^{17}$ For instance, in the case of humoral immunity, Lin et al. reported that IgM increased by $85 \%$ in the COVID-19 patients after 1-7 days of post-symptom onset (PSO). ${ }^{13}$ In addition, some studies reported increased IgA 1 day after onset of symptoms. ${ }^{13}$ However, IgG plays a more important role in the COVID-19 patients compared to $\operatorname{lgM}$ and IgA, as it is useful for recovery from the COVID-19 infection. ${ }^{18}$ Collectively, these data suggest that increase in titer of different classes of antibodies could be manifested during the COVID-19 infection in most immunocompetent individuals, and these last for several months.

Increasing studies of PID patients infected with COVID19 have provided striking information that could be contributed to a better understanding of COVID-19 infection. Quinti et al. have shown that patients with agammaglobulinemia presented a mild form of COVID-19, while patients with common variable immunodeficiency (CVID) manifested a severe form of the disease. ${ }^{19}$ In another study, Soresina et al. reported that two X-linked agammaglobulinemia (XLA) patients recovered from the SARS-CoV-2 infection without requirement of oxygen ventilation or intensive care but were susceptible to increased pneumonia after recovery from COVID-19. ${ }^{20}$ However, although some studies have supported the destructive role of B-cells in the SARS-CoV-2 infection, a study on XLA patient showed that the existence of functional B-cells would be necessary for viral clearance. ${ }^{21}$ Of note, several lines of evidence support the possibility of normalization of immunoglobulin levels during adulthood as more than $20 \%$ of patient with defined transient hypogammaglobulinemia diagnosis were identified in adult age. ${ }^{22}$ However, in the present study, transient increased levels of immunoglobulins to the normal range were seen during the COVID-19 infection, while after the resolution of infection, these levels reduced substantially to below normal range. This phenomenon was ambiguous for us, and it requires further investigation. 
The destructive role of inflammation induced by B-cells in the COVID-19 infection could be correlated with the different outcomes of COVID-19 infection between XLA and CVID patients, ${ }^{19,20,23}$ as agammaglobulinemia patients with the absence of B-cells manifested milder form of COVID-19 compared to CVID. This finding was promoted by Roschewski et al. ${ }^{24}$ Their study demonstrated that lung inflammatory responses and overproduction of macrophage-mediated cytokines were decreased in XLA patients following administration of acalabrutinib, which is a Bruton tyrosine kinase (BTK) blocker. ${ }^{24}$ Overall, to the complexity of mechanisms of the COVID-19 infection along with limitation of studies of PID patients, it is difficult to achieve exact clinical conclusions at this time. It seems, there is a long and winding road to solving this global pendemic.

\section{Conclusion}

We reported a case with HIgM phenotype and the COVID-19 infection. Given that coronavirus leads to cytokine storms (especially production of IL-6), it seems that IL-6 induces proliferation and production of B-cells in hypogammaglobulinemia patients in order to determine the role of this cytokine in terminal B-cell differentiation and IgG production. ${ }^{25}$ However, further studies are required to clarify the exact cause of transient increased levels of immunoglobulins during the COVID infection in immunodeficient patients.

\section{Conflict of interest}

The authors state that there are no conflicts of interest to disclose.

\section{References}

1. Hammarstrom $\mathrm{L}$ et al. Development of passive immunity against SARS-CoV-2 for management of immunodeficient patients-A perspective. J Allergy Clin Immunol. 2020;146(1):58-60. https://doi.org/10.1016/j.jaci.2020.04.043

2. Singhal T. A review of coronavirus disease-2019 (COVID-19). Indian J Pediatr. 2020;87(4):281-6. https://doi.org/10.1007/ s12098-020-03263-6

3. Rothan HA, Byrareddy SN. The epidemiology and pathogenesis of coronavirus disease (COVID-19) outbreak. J Autoimmun. 2020;109:102433. https://doi.org/10.1016/j.jaut.2020.102433

4. Zheng YY et al., COVID-19 and the cardiovascular system. Nat Rev Cardiol. 2020;17(5):259-60. https://doi.org/10.1038/ s41569-020-0360-5

5. Feng $\mathrm{S}$ et al. Rational use of face masks in the COVID-19 pandemic. Lancet Respir Med. 2020;8(5):434-6. https://doi. org/10.1016/S2213-2600(20)30134-X

6. Shi S et al. Association of cardiac injury with mortality in hospitalized patients with COVID-19 in Wuhan, China. JAMA Cardiol. 2020;5(7):802-10. https://doi.org/10.1001/jamacardio.2020.0950

7. Chan VW et al. A systematic review on COVID-19: Urological manifestations, viral RNA detection and special considerations in urological conditions. World J Urol. 2020:1-12. https://doi. org/10.1007/s00345-020-03246-4

8. Rodriguez-Morales AJ et al. Clinical, laboratory and imaging features of COVID-19: A systematic review and metaanalysis. Travel Med Infect Dis. 2020;34:101623. https://doi. org/10.1016/j.tmaid.2020.101623

9. Terpos $\mathrm{E}$ et al. Hematological findings and complications of COVID-19. Am J Hematol. 2020;95(7):834-47. https://doi. org/10.1002/ajh.25829

10. Tang YW et al. Laboratory diagnosis of COVID-19: Current issues and challenges. J Clin Microbiol. 2020;58(6):e00512-20. https://doi.org/10.1128/JCM.00512-20

11. Li Y, Xia L. Coronavirus disease-2019 (COVID-19): Role of chest $\mathrm{CT}$ in diagnosis and management. AJR Am J Roentgenol. 2020;214(6):1280-6. https://doi.org/10.2214/AJR.20.22954

12. Fang $Y$ et al. Sensitivity of chest CT for COVID-19: Comparison to RT-PCR. Radiology. 2020;296(2):e115-7. https://doi.org/ 10.1148/radiol.2020200432

13. Lin $\mathrm{D}$ et al. Evaluations of the serological test in the diagnosis of 2019 novel coronavirus (SARS-CoV-2) infections during the COVID-19 outbreak. Eur J Clin Microbiol Infect Dis. 2020;39:17. https://doi.org/10.1007/s10096-020-03978-6

14. Lisboa Bastos $M$ et al. Diagnostic accuracy of serological tests for COVID-19: Systematic review and meta-analysis. BMJ. 2020;370:m2516. https://doi.org/10.1136/bmj.m2516

15. $\mathrm{Xu} \mathrm{X}$ et al. Effective treatment of severe COVID-19 patients with tocilizumab. Proc Natl Acad Sci USA. 2020;117(20):1097075. https://doi.org/10.1073/pnas.2005615117

16. Qamar N, Fuleihan RL. The hyper-lgM syndromes. Clin Rev Allergy Immunol. 2014;46(2):120-30. https://doi.org/10.1007/ s12016-013-8378-7

17. Cañete PF, Vinuesa CG. COVID-19 makes B cells forget, but T cells remember. Cell. 2020;183(1):13-15. https://doi.org/10.1016/ j.cell.2020.09.013

18. Theel ES et al. The role of antibody testing for SARS-CoV-2: Is there one? J Clin Microbiol. 2020;58(8): e00797-20. https:// doi.org/10.1128/JCM.00797-20

19. Quinti I et al. A possible role for B cells in COVID-19? Lesson from patients with agammaglobulinemia. J Allergy Clin Immunol. 2020;146(1):211-3.e4. https://doi.org/10.1016/j. jaci.2020.04.013

20. Soresina A et al. Two X-linked agammaglobulinemia patients develop pneumonia as COVID-19 manifestation but recover. Pediatr Allergy Immunol. 2020;31(5):565-9. https://doi. org/10.1111/pai.13263

21. Mira E et al. Rapid recovery of a SARS-CoV-2-infected X-linked agammaglobulinemia patient after infusion of COVID-19 convalescent plasma. J Allergy Clin Immunol Pract. 2020;8(8):27935. https://doi.org/10.1016/j.jaip.2020.06.046

22. Ameratunga $\mathrm{R}$ et al. Transient hypogammaglobulinaemia of infancy: Many patients recover in adolescence and adulthood. Clin Exp Immunol. 2019;198(2):224-32. https://doi.org/10.1111/ cei.13345

23. Babaha F, Rezaei N. Primary immunodeficiency diseases in COVID-19 pandemic: A predisposing or protective factor? Am J Med Sci. 2020; https://doi.org/10.1016/j.amjms.2020.07.027

24. Roschewski $M$ et al. Inhibition of bruton tyrosine kinase in patients with severe COVID-19. Sci Immunol. 2020;5(48). https://doi.org/10.1126/sciimmunol.abd0110

25. Maeda $\mathrm{K}$ et al. IL-6 increases B-cell IgG production in a feed-forward proinflammatory mechanism to skew hematopoiesis and elevate myeloid production. Blood. 2010;115(23):4699706. https://doi.org/10.1182/blood-2009-07-230631 